\title{
Synergy of interleukin-4 and interferon- $\gamma$ in arginase-1 production in RAW264.7 macrophages
}

\author{
Tomoko Hinse Endo, Natsumi Mizuno, Saeko Matsuda, Saki Shiga, Yoshiki Yanagawa
}

\begin{abstract}
Background: Interferon (IFN) $-\gamma$ and interleukin (IL)- 4 are considered to be important factors to regulate immune responses. Although the effects of IFN- $\gamma$ or IL- 4 on macrophage functions are well established, their cooperative action is not fully understood.
\end{abstract}

Objective: Inducible nitric oxide synthase (iNOS) or arginase (Arg)-1 is a representative marker of M1 or M2 macrophages and plays a role in the acceleration or suppression of inflammatory responses. In the present study, we examined the effect of simultaneous treatment with IFN- $\gamma$ and IL-4 on macrophage expression of iNOS and Arg-1 using the murine macrophage cell line RAW264.7.

Methods: Protein production and mRNA expression of iNOS and Arg-1 were measured using immunoblotting and reverse transcription-polymerase chain reaction. Cell surface expression of CD86 and programmed death ligand (PD-L) 2 was analyzed using flow cytometry.

Results: IFN- $\gamma$ or IL-4 increased iNOS or Arg-1 protein production, respectively. Of note, IL- 4 combined with IFN- $\gamma$ synergistically increased Arg-1 protein production, whereas IL-4 inhibited IFN- $\gamma$-induced iNOS production. This phenomenon was consistent with the mRNA levels. In addition, IL-4 combined with IFN- $\gamma$ synergistically increased cell surface expression of PD-L2, which is involved in T cell suppression, whereas IL- 4 completely inhibited IFN- $\gamma$-induced expression of CD86, which is responsible for $\mathrm{T}$ cell activation.

Conclusion: In the present study, we found the synergy of IFN- $\gamma$ and IL-4 in Arg-1 and PD-L2 expression. Thus, macrophages highly expressing Arg-1 and PD-L2 may be induced by both IFN- $\gamma$ and IL-4 at the inflammatory site, and might play a role in the regulation of inflammatory immune responses.

Key words: Arginase-1, interleukin-4, interferon- $\gamma$, macrophages, programmed death ligand 2

\section{From:}

Department of Pharmacology, School of Pharmaceutical Sciences, Health Sciences University of Hokkaido, Hokkaido, Japan

\author{
Corresponding author: \\ Yoshiki Yanagawa \\ Department of Pharmacology, School of Pharmaceutical Sciences, \\ Health Sciences University of Hokkaido, \\ 1757 Kanazawa, Ishikari-Tobetsu, Hokkaido 061-0293, Japan \\ E-mail: yanagawa@hoku-iryo-u.ac.jp
}

\section{Introduction}

Interferon (IFN)- $\gamma$, a representative $\mathrm{T}$ helper (Th) type 1 cytokine, plays a decisive role in Th1 polarization, which promotes cell-mediated immunity involving NK cells and $\mathrm{CD}^{+}$ $\mathrm{T}$ cells, ultimately leading to the elimination of intracellular pathogens and tumor cells. ${ }^{1}$ IFN- $\gamma$ also plays a pathogenic role in several autoimmune diseases, including type 1 diabetes, multiple sclerosis, and rheumatoid arthritis., ${ }^{2,3}$ In contrast, it has been suggested that IFN- $\gamma$ not only exhibits immunostimulatory properties but also contributes to anti-inflammatory immune responses in certain animal models of autoimmune disease. ${ }^{4-6}$ However, the paradoxical roles of IFN- $\gamma$ are not fully understood.

Interleukin (IL)-4, mainly produced by activated Th2 cells, is responsible for Th2 polarization and humoral immunity involving B cells. ${ }^{1}$ IL-4 promotes the differentiation of B cells to plasma cells, thereby inducing the production of antibodies to eradicate extracellular pathogens. However, IL-4 plays a pathogenic role in allergic diseases including asthma and 
atopic dermatitis. ${ }^{7}$ IL-4 receptor is expressed by macrophages and IL-4 signals appear to regulate macrophage functions. It was reported that IL- 4 inhibits macrophage production of IL12 , a potent IFN- $\gamma$ inducer. $^{8}$ In addition, IL- 4 interferes with IFN- $\gamma$ receptor signaling, ${ }^{9}$ whereas IFN- $\gamma$ silences T cell expression of IL-4, during Th1 or Th2 differentiation. ${ }^{10}$ Thus, IL- 4 appears to negatively regulate IFN- $\gamma$-mediated immune responses.

Macrophages play a crucial role in innate and adaptive immune responses and are also involved in maintenance of tissue homeostasis. Macrophages can be divided into two groups, namely, M1 macrophages (classical macrophages) and M2 macrophages (alternatively activated macrophages), based on their functions. ${ }^{11,12}$ M1 macrophages are induced by stimulation with Th1 cytokines and bacterial components and are responsible for the acceleration of inflammatory immune responses. IFN- $\gamma$ is a potent inducer of M1 macrophages and increases the macrophage production of inflammatory mediators, such as tumor necrosis factor (TNF)- $\gamma$ and inducible nitric oxide synthase (iNOS). iNOS is a representative marker of M1 macrophages and iNOS-derived nitric oxide (NO) plays a role in inflammation. In contrast, M2 macrophages are generated by stimulation with Th2 cytokines and immune regulatory mediators and are involved in the suppression of inflammation and acceleration of tissue repair. ${ }^{11-14}$ IL-4 is a typical stimulator that generates M2 macrophages and induces the production of arginase (Arg)-1. Arg-1 is a representative marker of M2 macrophages and is responsible for tissue repair. Arg-1 deficiency delays wound healing with concomitant increased infiltration of $\mathrm{iNOS}^{+}$cells into the wound site, resulting in excessively prolonged inflammation. ${ }^{15}$ Therefore, Arg-1 is responsible for M2 macrophage functions and the balance of iNOS and Arg-1 expression is crucial to regulate inflammation and tissue repair for tissue homeostasis.

Although the effects of IFN- $\gamma$ or IL-4 on macrophage functions are well established, their cooperative action is not fully understood. In the present study, we examined the effect of simultaneous treatment with IFN- $\gamma$ and IL-4 on macrophage expression of iNOS and Arg-1 using the murine macrophage cell line RAW264.7.

\section{Methods \\ Reagents and antibodies (Abs)}

RPMI-1640 medium was obtained from Sigma-Aldrich (Saint Louis, MO, USA). Fetal bovine serum (FBS) and penicillin-streptomycin were purchased from Thermo Fisher Scientific Inc. (Waltham, MA, USA). Recombinant murine IFN- $\gamma$ and IL-4 were obtained from PeproTech (Cranbury, NJ, USA). These cytokines were used at $40 \mathrm{ng} / \mathrm{ml}$, because our preliminary dose-response study indicated that both cytokines exerted maximum effects at this concentration.

Anti-signal transducer and activator of transcription (STAT) 6 monoclonal Ab (mAb, 16G12A08) and anti-Arg-1 $\mathrm{mAb}$ (O94E6/ARG1) were acquired from BioLegend (San Diego, CA, USA). Anti-iNOS mAb (6/iNOS/NOS Type II) and anti-phospho-STAT6 (Y641) mAb (J71-773.58.11) were obtained from BD Biosciences (Franklin Lakes, NJ, USA). Anti-STAT1 Ab, anti-phospho-STAT1 (Tyr701) mAb (58D6), anti-glyceraldehyde-3-phosphate dehydrogenase (GAPDH) mAb
(14C10), and anti-rabbit IgG horseradish peroxidase (HRP)linked $\mathrm{Ab}$ were purchased from Cell Signaling Technology (Beverly, MA, USA).

Phycoerythrin (PE)-conjugated anti-mouse CD86 mAb (GL-1) and allophycocyanin (APC)-conjugated anti-mouse CD80 mAb (16-10A1) were purchased from BioLegend. $\mathrm{PE}$-conjugated anti-mouse programmed death ligand (PD-L) 2 (TY25) and APC-conjugated anti-mouse PD-L1 (MIH5) were obtained from BD Biosciences. PE- or APC-conjugated isotype-matched control mAbs were acquired from BioLegend and BD Biosciences.

\section{Cell culture}

RAW264.7 cells were obtained from the American Type Culture Collection (ATCC, Manassas, VA, USA) and cultured in RPMI-1640 with 5\% FBS, $100 \mathrm{IU} / \mathrm{ml}$ of penicillin, and 100 $\mu \mathrm{g} / \mathrm{ml}$ of streptomycin.

\section{Measurement of protein production using immunoblotting}

RAW264.7 cells were cultured with or without IFN- $\gamma$ (40 $\mathrm{ng} / \mathrm{ml})$ and/or IL-4 $(40 \mathrm{ng} / \mathrm{ml})$ for the indicated times at a density of $5 \times 10^{5} \mathrm{cells} / \mathrm{ml} /$ well or $1 \times 10^{6}$ cells $/ \mathrm{ml} /$ well using a low-adhesion surface 24-well plate, EZ-BindShut ${ }^{\mathrm{Tn}}$ SP (AGC TECHNO GLASS Co., Ltd. Shizuoka, Japan). The reactions were halted by rapidly cooling on ice and the cells were collected. The cells were washed with ice-cold phosphate-buffered saline, and whole-cell lysates were prepared using cell lysis buffer (Cell Signaling Technology). The cell lysates were separated using SDS-PAGE and then blotted onto a polyvinylidene fluoride membrane (Millipore, Bedford, MA, USA). The membrane was probed with a primary $\mathrm{Ab}$ and developed using a HRP-linked secondary Ab via enhanced chemiluminescence. The luminescence intensity was quantified using a luminescent image analyzer, ImageSaver6 (ATTO Corporation, Tokyo, Japan), and a software program, CS Analyzer 3 (ATTO Corporation). The intensity relative to the mean of all the band intensities in each experiment is shown as a relative intensity. ${ }^{16}$

\section{Quantification of $m R N A$ expression using reverse transcrip- tion (RT)-polymerase chain reaction (PCR)}

RAW264.7 cells were cultured with or without IFN- $\gamma$ (40 $\mathrm{ng} / \mathrm{ml})$ and/or IL-4 $(40 \mathrm{ng} / \mathrm{ml})$ for the indicated times at a density of $1 \times 10^{6}$ cells $/ \mathrm{ml} /$ well in a 24 -well plate (EZ-BindShut $^{\mathrm{TM}}$ SP). Total RNA was isolated from the RAW264.7 cells using TRIzol reagent (Thermo Fisher Scientific, Waltham, MA, USA). The mRNA levels of iNOS, Arg- 1 , TNF- $\alpha$, IL- $1 \beta$, PD-L2, and GAPDH in the RNA samples were determined through quantitative RT-PCR using the TB Green ${ }^{\oplus}$ Premix Ex Taq $^{\text {TM }}$ II (Takara Bio Inc., Shiga, Japan), as described elsewhere. ${ }^{17}$ PCR was performed using the 7500 Real-Time PCR system or StepOnePlus Real ${ }^{\mathrm{TM}}$ Time PCR System (Applied Biosystems, Waltham, MA, USA). Melt curve analysis and the fragment size of the PCR products were used to verify gene-specific amplification. The primer sequences and amplicon sizes were as follows: iNOS forward, 5'-GTTCTCAGCC CAACAATACAAGA-3' and reverse, 5'-GTGGACGGGTCG ATGTCAC-3' (126 bp); Arg-1 forward, 5'-CTCCAAGCCAA AGTCCTTAGAG-3' and reverse, 5'-AGGAGCTGTCATTAG 
GGACATC-3' (184 bp); TNF- $\alpha$ forward, 5' -CCCTCACACTC AGATCATCTTCT-3' and reverse, 5' -GCTACGACGTGGGC TACAG-3' (61 bp); IL-1 $\beta$ forward, 5'-GCAACTGTTCCTGA ACTCAACT-3' and reverse, 5' ${ }^{\prime}$-ATCTTTTGGGGTCCGTCA ACT-3' (88 bp); PD-L2 forward, 5'-GAGCCAGTTTGCAGA AGGTAG-3' and reverse, 5' -ATCCGACTCAGAGGGTCAAT G-3' (471 bp); IL-4 receptor a (IL4R) forward, 5' - TCTGC ATCCCGTTGTTTTGC-3' and reverse, 5'- GCACCTGTGC ATCCTGAATG-3' (128 bp); GAPDH forward, 5'-AGGTCG GTGTGAACGGATTTG-3' and reverse, 5' -TGTAGACCATG TAGTTGAGGTCA-3' (122 bp).

\section{Measurement of the cell surface expression of CD80, CD86, PD-L1, and PD-L2}

RAW264.7 cells were cultured with or without IFN- $\gamma$ (40 $\mathrm{ng} / \mathrm{ml})$ and/or IL-4 (40 ng/ml) for 20 hours (hr) at a density of $5 \times 10^{5}$ cells $/ \mathrm{ml} /$ well in a 24 -well plate (EZ-BindShut ${ }^{\mathrm{Tm}}$ SP). The cells were collected and incubated with anti-CD16/32 $\mathrm{mAb}$ (BioLegend) to prevent binding of specific $\mathrm{mAb}$ to the $\mathrm{Fc} \gamma$ III/II receptor. Subsequently, the cells were stained with $\mathrm{PE}$ - or APC-conjugated mAbs. Isotype-matched control $\mathrm{mAb}$ was used as a negative control. The expression of cell surface markers was analyzed using flow cytometry on Accuri ${ }^{\mathrm{Tm}} \mathrm{C6}$ (BD Biosciences).

\section{Measurement of TNF- $\alpha$ production using enzyme-linked im- munosorbent assay (ELISA)}

RAW264.7 cells were cultured with or without IFN- $\gamma(40$ $\mathrm{ng} / \mathrm{ml})$ and/or IL-4 (40 ng/ml) for $24 \mathrm{hr}$ at a density of $5 \times 10^{5}$ cells $/ \mathrm{ml} /$ well in a 24-well plate (EZ-BindShut ${ }^{\mathrm{TM}} \mathrm{SP}$ ). Culture supernatant was collected and TNF- $\alpha$ levels were measured using an ELISA kit (BioLegend).

\section{Nitric oxide (NO) measurement}

RAW264.7 cells were cultured with or without IFN- $\gamma(40$ $\mathrm{ng} / \mathrm{ml})$ and/or IL-4 (40 ng/ml) for the indicated times at a density of $5 \times 10^{5}$ cells $/ \mathrm{ml} /$ well in a 24 -well plate (EZ-BindShut $^{\text {tix }}$ SP). The supernatants $(100 \mu \mathrm{l})$ were added to $100 \mu \mathrm{l}$ of $6 \mathrm{mg} / \mathrm{ml}$ Griess-Romijin reagent and incubated at room temperature for 10 minutes (min). The absorbance at $540 \mathrm{~nm}$ was measured using a microplate reader. Nitrite concentrations were calculated using a sodium nitrite standard curve as a reference.

\section{Statistical analysis}

Data are expressed as the mean \pm standard error of the mean. The data were analyzed using one-way analysis of variance followed by Tukey's test to discriminate significant differences. The level of significance was set at $p<0.05$.

\section{Results}

Effects of IFN- $\gamma$ and IL-4 on iNOS and Arg-1 protein production

iNOS or Arg-1 is a representative marker of M1 or M2 macrophages. ${ }^{11}$ We examined the effect of IFN- $\gamma$, IL- 4 , or simultaneous treatment with these two cytokines on iNOS and Arg-1 protein production in RAW264.7 macrophages. The cells were stimulated with IFN- $\gamma$, IL-4, or both for 24,48 , and $72 \mathrm{hr}$ and the protein levels of iNOS and Arg-1 in the cells were determined using immune blotting. IFN- $\gamma$ markedly increased iNOS production at 24 and $48 \mathrm{hr}$, and the IFN- $\gamma$-induced iNOS level decreased at $72 \mathrm{hr}$ compared to that in the prior periods (Figure 1A and B). IL-4 alone showed no effect on iNOS protein level, whereas IL-4 inhibited IFN- $\gamma$-induced iNOS production. Although IFN- $\gamma$ or IL- 4 alone showed

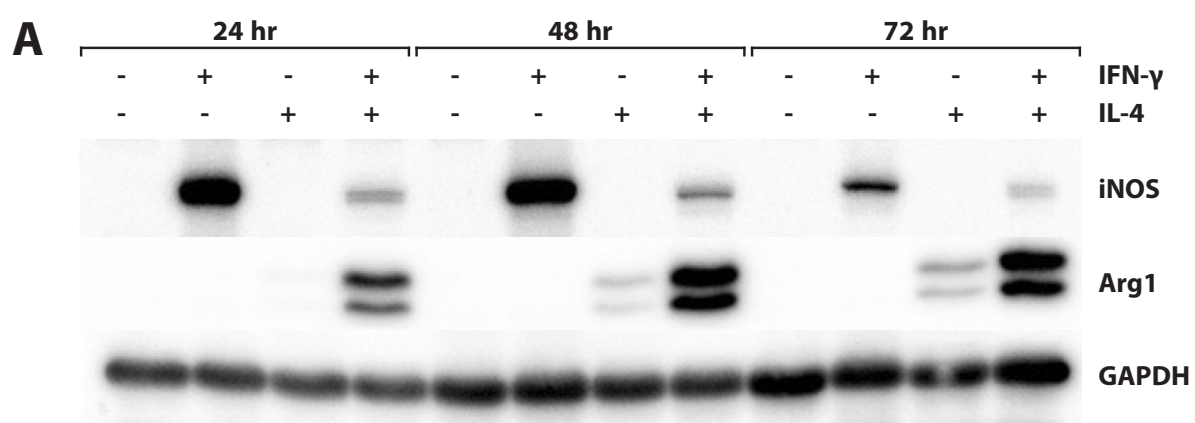

Figure 1. Effects of interferon (IFN) $-\gamma$ and interleukin (IL)-4 on the production of inducible nitric synthase (iNOS) and arginase (Arg)-1 in RAW264.7 macrophages. RAW264.7 macrophages were stimulated with IFN- $\gamma$ (40 ng/ml), IL-4 (40 ng/ml), or both for 24, 48, or 72 hours. Intracellular protein levels of iNOS, Arg-1, and glyceraldehyde-3-phosphate dehydrogenase (GAPDH) were determined using immunoblotting. (A) A representative immunoblot is presented. The relative intensity of the specific band for iNOS (B) and Arg-1 (C) is shown. (D) Nitric oxide (NO) concentration in the culture supernatant was measured using Griess-Romijin reagent. Each column represents the mean \pm standard error of the mean of five independent experiments. Statistical significance was calculated using Tukey's test $\left({ }^{\star} p<0.05,{ }^{* *} p<0.01,{ }^{* *} p<0.001\right)$. 
B

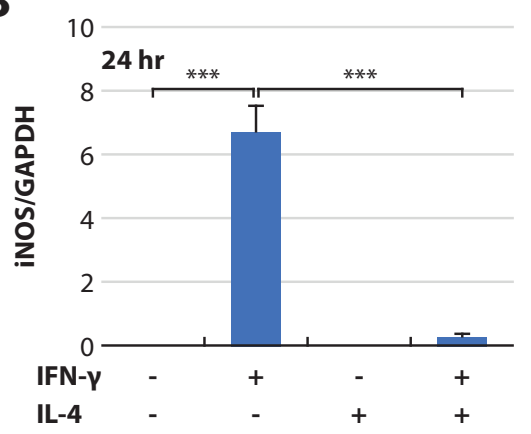

C

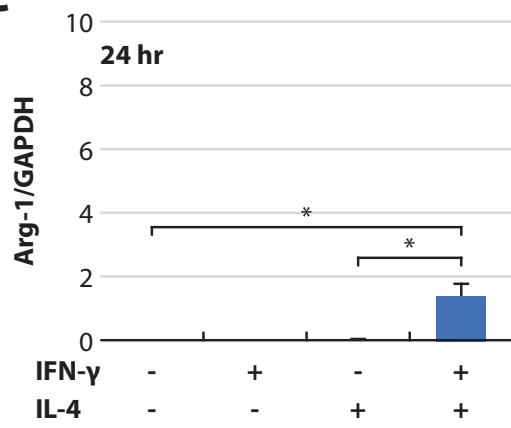

D

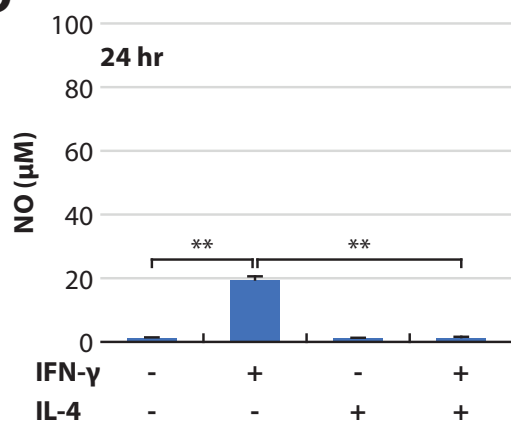

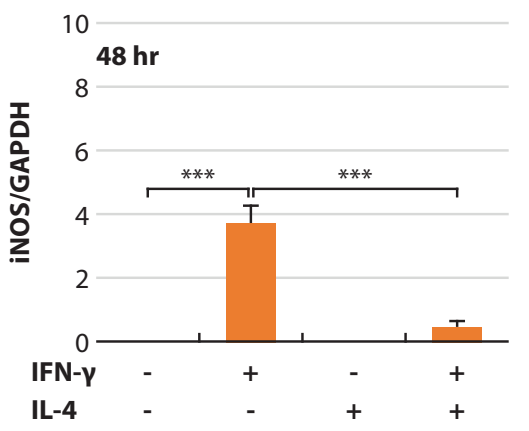
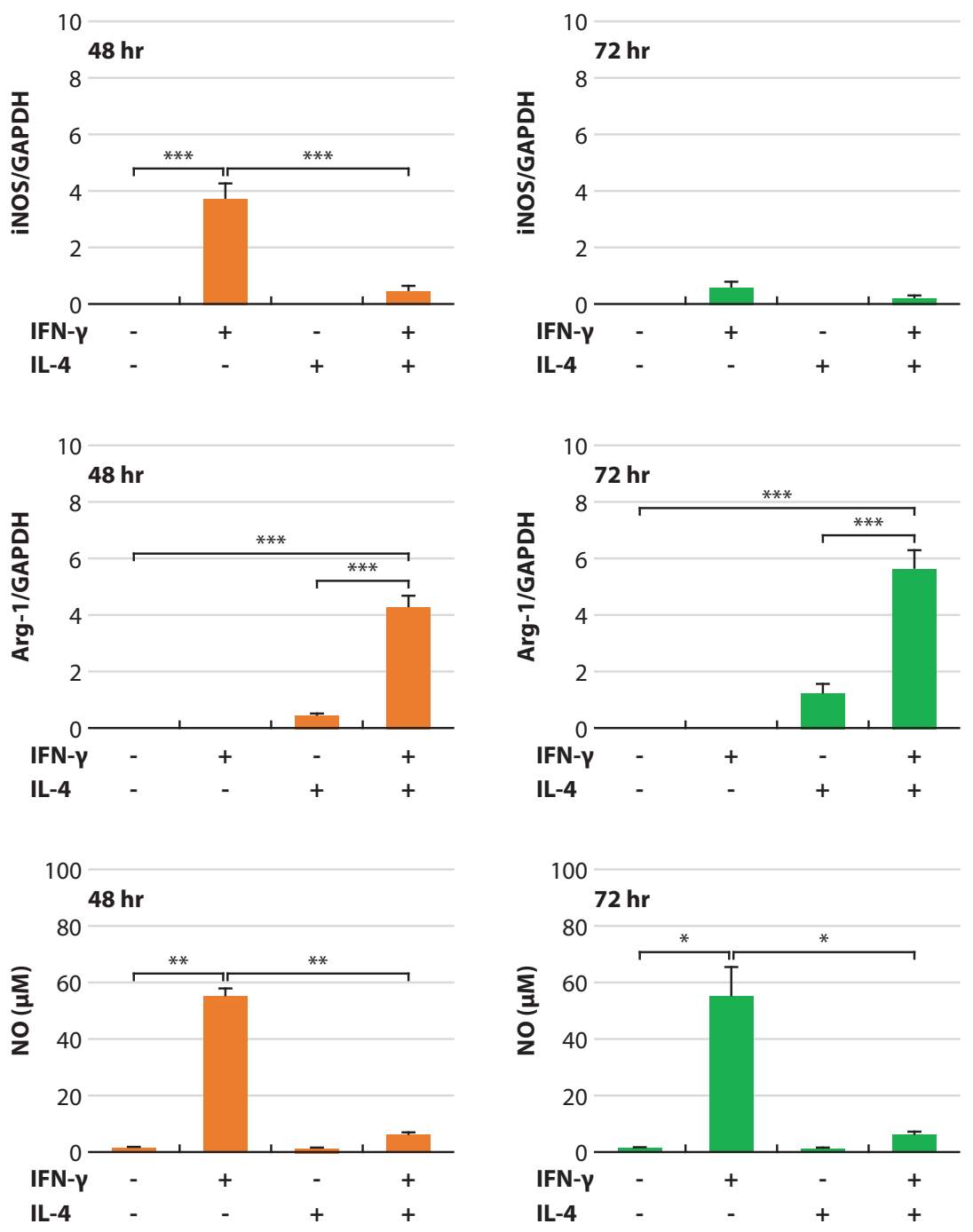

Figure 1. (Continued)

little or no effect on Arg-1 production at $24 \mathrm{hr}$, IL-4 combined with IFN- $\gamma$ significantly increased Arg-1 production (Figure $\mathbf{1 A}$ and $\mathrm{C}$, left). IL-4 alone could induce modest production of Arg-1 at 48 and $72 \mathrm{hr}$ (Figure 1A and C, center and right). Of note, IL- 4 combined with IFN- $\gamma$ remarkably increased Arg-1 production at these time points.

We also examined the effects of IFN- $\gamma$ and IL-4 on NO production by RAW264.7 macrophages. IFN- $\gamma$ significantly increased NO production at 24, 48, and $78 \mathrm{hr}$ (Figure 1D). IL-4 alone showed no effect on NO production at the tested time points. However, IL-4 significantly suppressed IFN- $\gamma$-induced NO production.

\section{Effects of IFN- $\gamma$ and IL-4 on expression of mRNA for iNOS and Arg-1}

We examined the effects of IFN- $\gamma$, IL-4, or simultaneous treatment with these two cytokines on iNOS and Arg-1 mRNA expression in RAW264.7 macrophages. The cells were stimulated with IFN- $\gamma$, IL-4, or both for 4,8 , and $16 \mathrm{hr}$ and the mRNA levels of iNOS and Arg-1 in the cells were analyzed using quantitative RT-PCR. IFN- $\gamma$ alone induced iNOS mRNA expression modestly at 4 and $8 \mathrm{hr}$ and markedly at
$16 \mathrm{hr}$ (Figure 2A). IL-4 alone showed no effects on iNOS mRNA expression at all the tested time points. However, IL-4 inhibited IFN- $\gamma$-induced iNOS mRNA expression.

Arg-1 mRNA levels were increased by treatment with IL-4 at all the tested time points (Figure 2B). Although IFN- $\gamma$ alone failed to increase Arg- 1 mRNA levels, IFN- $\gamma$ combined with IL-4 synergistically induced Arg-1 mRNA expression.

\section{Effects of IFN- $\gamma$ and IL-4 on the expression of inflammatory cytokines}

Macrophages produce inflammatory cytokines in response to various inflammatory stimuli including IFN- $\gamma$. We next examined the effects of IFN- $\gamma$, IL-4, or simultaneous treatment with these cytokines on mRNA expression of inflammatory cytokines, TNF- $\alpha$ and IL-1 $\beta$, in RAW264.7 macrophages. The cells were stimulated with IFN- $\gamma$, IL- 4 , or both for $4 \mathrm{hr}$ and the mRNA levels of TNF- $\alpha$ and IL- $1 \beta$ in the cells were analyzed using quantitative RT-PCR. IFN- $\gamma$ markedly increased the levels of mRNA for TNF- $\alpha$ and IL- $1 \beta$, whereas the IFN- $\gamma$ induced increase in these cytokines was completely inhibited by treatment with IL-4 (Figure 3A). 
A

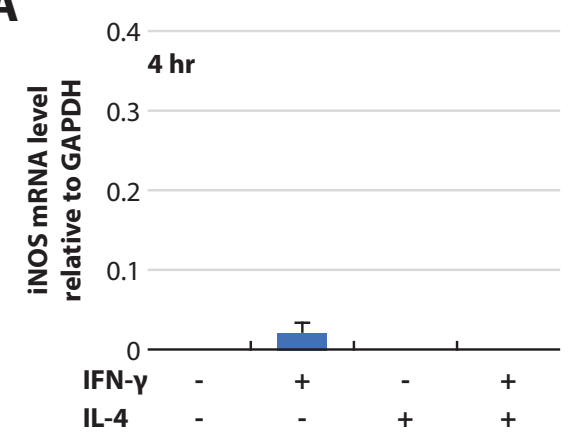

B

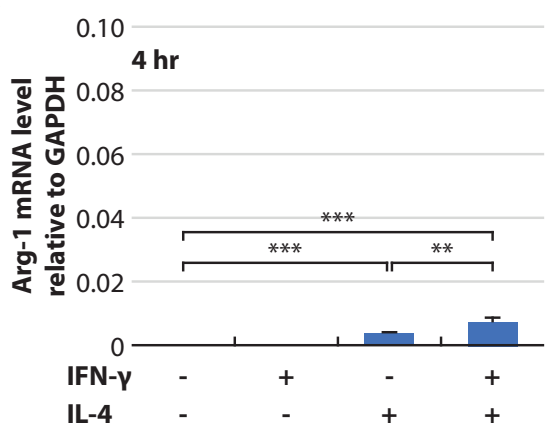

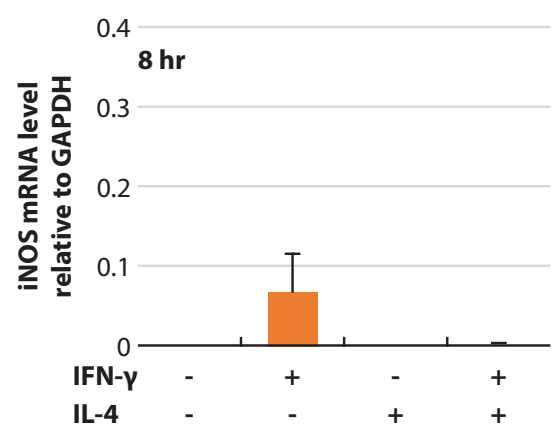
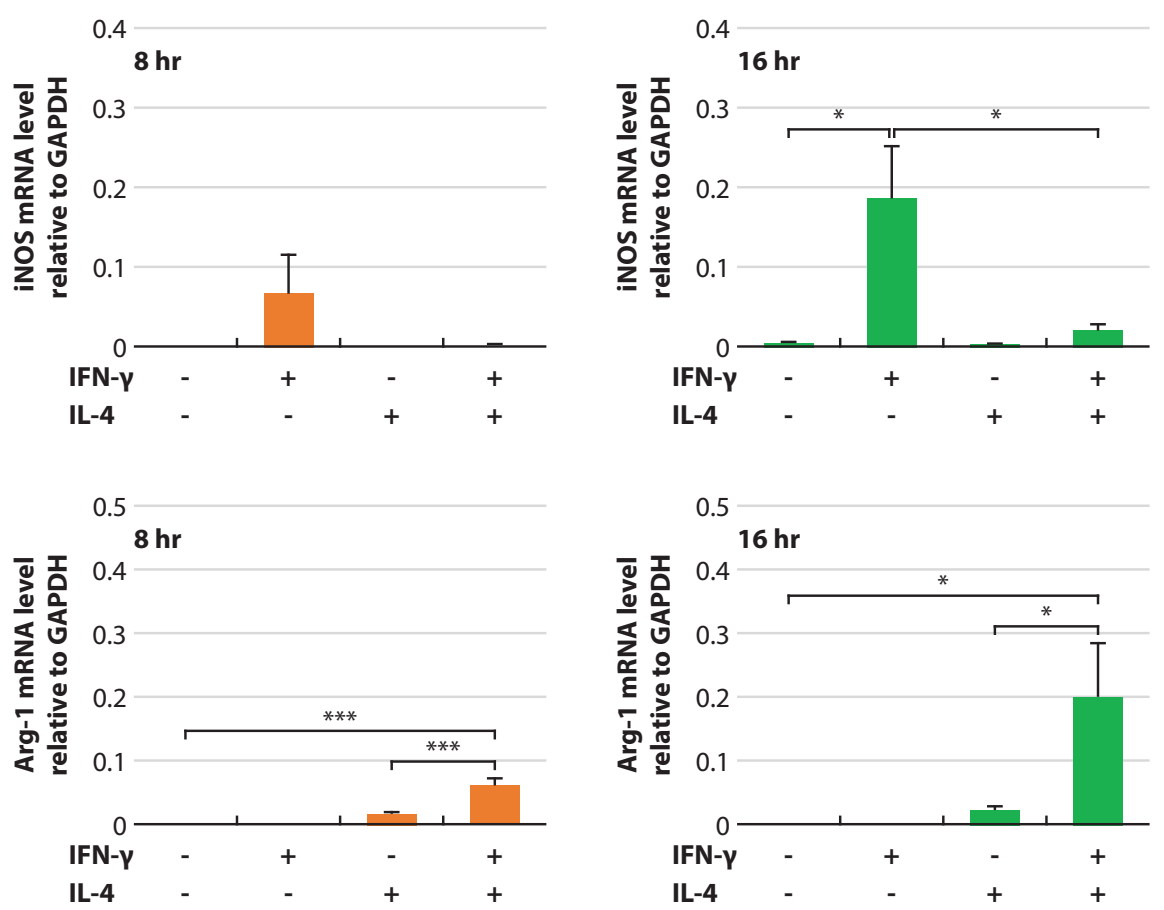

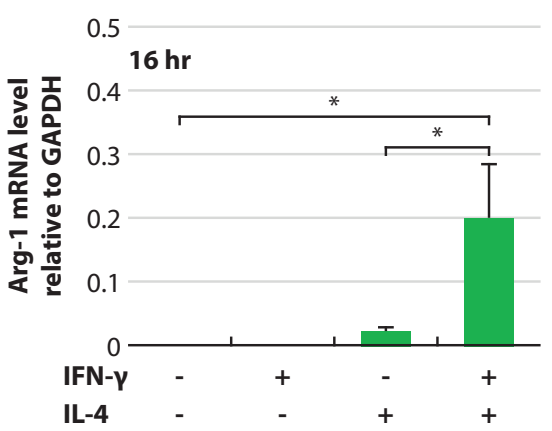

Figure 2. Effects of interferon (IFN)- $\gamma$ and interleukin (IL)-4 on mRNA expression of inducible nitric synthase (iNOS) and arginase (Arg)-1 in RAW264.7 macrophages. RAW264.7 macrophages were stimulated with IFN- $\gamma$ (40 ng/ml), IL-4 (40 ng/ $\mathrm{ml}$ ), or both for 4, 8, and 16 hours followed by total RNA isolation. The level of iNOS, Arg-1, and glyceraldehyde-3-phosphate dehydrogenase (GAPDH) mRNA expression in the total RNA was analyzed using quantitative reverse transcription-polymerase chain reaction. The mRNA level of iNOS (A) and Arg-1 (B) relative to the GAPDH gene. Each column presents the mean \pm standard error of the mean of eight independent experiments. Statistical significance was calculated using Tukey's test $\left({ }^{*} p<0.05,{ }^{* *} p<0.01,{ }^{* * *} p<0.001\right)$.

A

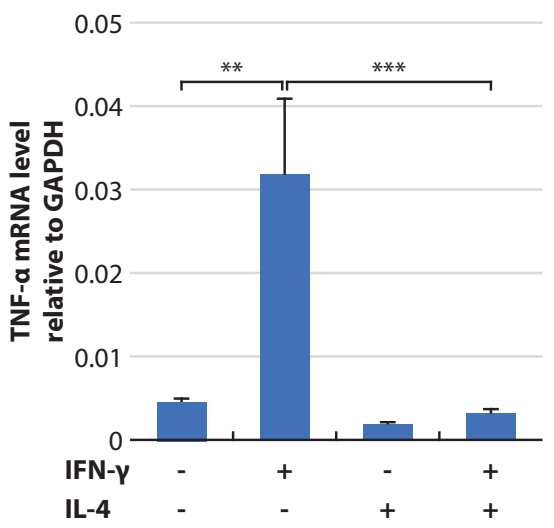

0.15

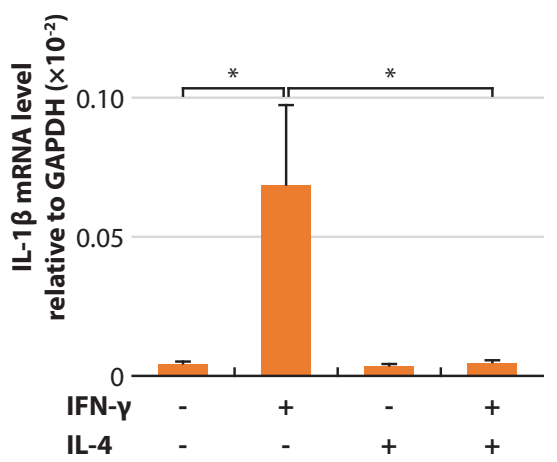

B

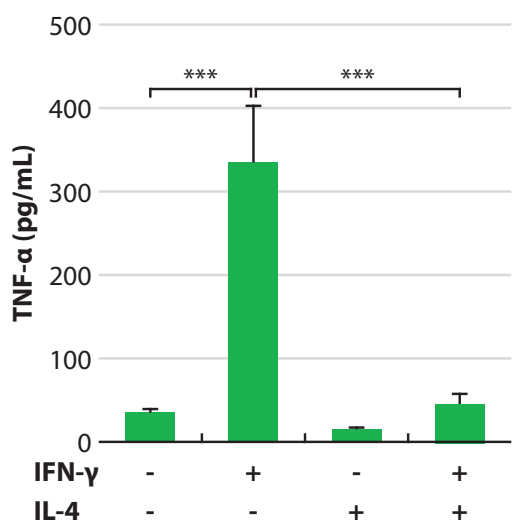

Figure 3. Effect of interferon (IFN) $-\gamma$ and interleukin (IL)-4 on tumor necrosis factor (TNF)- $\alpha$ and IL-1 $\beta$ expression in RAW264.7 macrophages. RAW264.7 macrophages were stimulated with IFN- $\gamma$ (40 ng/ml), IL-4 (40 ng/ml), or both. (A) At 4 hours after the stimulation, the total RNA of the cells was isolated and the level of TNF- $\alpha$, IL-1 $\beta$, and glyceraldehyde-3-phosphate dehydrogenase (GAPDH) mRNA expression in the RNA samples was analyzed using quantitative reverse transcription-polymerase chain reaction. mRNA levels of TNF- $\alpha$ and IL-1 $\beta$ relative to that of GAPDH are shown. Each column represents the mean \pm standard error of the mean of eight independent experiments. (B) At 24 hours after the stimulation, the protein level of TNF- $\alpha$ in the culture supernatant was measured using enzyme-linked immunosorbent assay. Each column represents the mean \pm standard error of the mean of four independent experiments. Statistical significance was calculated using Tukey's test $\left({ }^{*} p<0.05,{ }^{* *} p<0.01,{ }^{* *} p<0.001\right)$. 
We also measured the protein level of TNF- $\alpha$ in the culture supernatant at $24 \mathrm{hr}$ using ELISA. IFN- $\gamma$ significantly increased the amount of TNF- $\alpha$ protein production, whereas IFN- $\gamma$-induced TNF- $\alpha$ production was almost completely inhibited by treatment with IL-4 (Figure 3B).

\section{Effects of IFN- $\gamma$ and IL-4 on CD80 and CD86 expression}

Costimulatory molecules, CD80 and CD86, are expressed on antigen-presenting cells, including macrophages, and play a crucial role in activating $\mathrm{T}$ cells during antigen presentation. ${ }^{18}$ In addition, CD80 and CD86 are recognized as markers of M1 macrophages. ${ }^{11}$ We thus examined the effects of IFN- $\gamma$ and IL-4 on the expression of these costimulatory molecules in RAW264.7 macrophages. The cells were stimulated with IFN- $\gamma$, IL-4, or both for $20 \mathrm{hr}$ and cell surface expression of CD80 and CD86 was analyzed using flow cytometry. CD80 ${ }^{+}$ and $\mathrm{CD}^{+} 6^{+}$cells were gated based on the negative control with isotype-matched control mAb. RAW264.7 cells spontaneously expressed CD80 and CD86 in the culture with medium alone. As expected, IFN- $\gamma$ significantly increased CD80 and CD86 expression (Figure 4A and B). IL-4 alone showed no effect on the expression of these molecules. However, IL-4 completely inhibited IFN- $\gamma$-induced CD86 expression (Figure 4B right).
Although IL-4 also inhibited IFN- $\gamma$-induced CD80 expression, its effect was not statistically significant (Figure 4B left).

\section{Effects of IFN- $\gamma$ and IL-4 on PD-L1 and PD-L2 expression}

Immune checkpoint molecules, PD-L1 and PD-L2, on antigen-presenting cells are involved in $\mathrm{T}$ cell suppression. ${ }^{19} \mathrm{It}$ has been reported that PD-L2 expression is correlated with Arg-1 expression in alternatively activated macrophages. ${ }^{20}$ We thus examined the effects of IFN- $\gamma$ and IL-4 on PD-L2 expression in RAW264.7 macrophages. The cells were stimulated with IFN- $\gamma$, IL-4, or both for $20 \mathrm{hr}$ and cell surface expression of PD-L1 and PD-L2 was analyzed using flow cytometry. IFN- $\gamma$ markedly increased the surface expression of PD-L1, but not PD-L2 (Figure 5A and B). IL-4 moderately increased the expression of these molecules and showed no effect on IFN- $\gamma$-induced PD-L1 expression (Figure 5B, left). Interestingly, simultaneous treatment with IFN- $\gamma$ and IL- 4 synergistically increased the expression of PD-L2 (Figure 5B, right).

We also analyzed PD-L2 mRNA expression in RAW264.7 cells at $4 \mathrm{hr}$ (Figure $5 \mathrm{C}$ ). IFN- $\gamma$ failed to increase PD-L2 mRNA expression, while IL-4 modestly increased PD-L2 mRNA expression. In contrast, IL-4 combined with IFN- $\gamma$ synergistically increased PD-L2 mRNA expression.

A
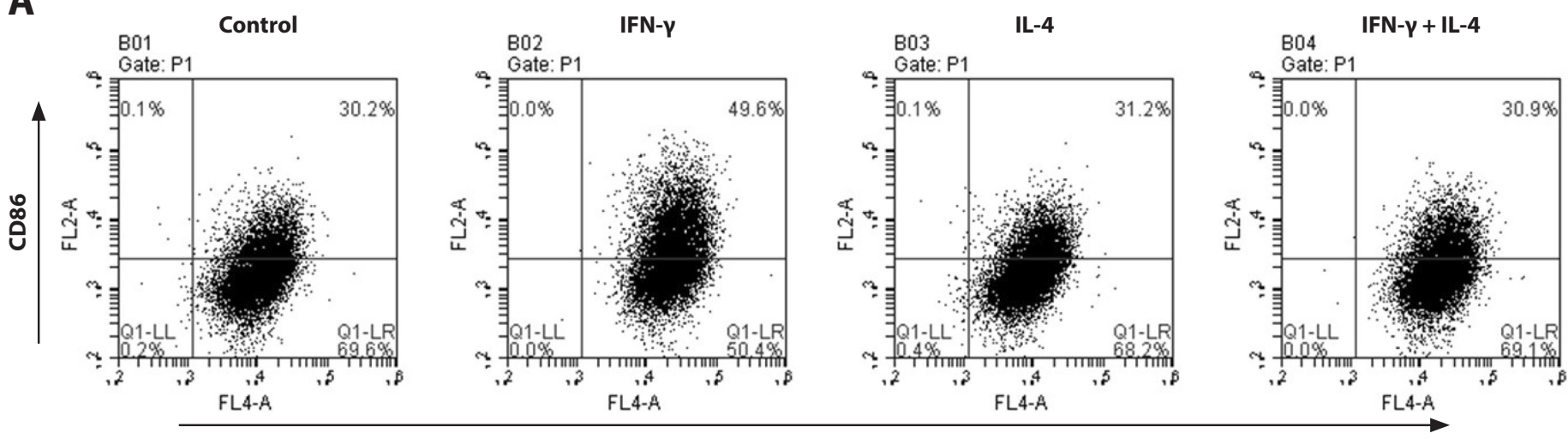

B
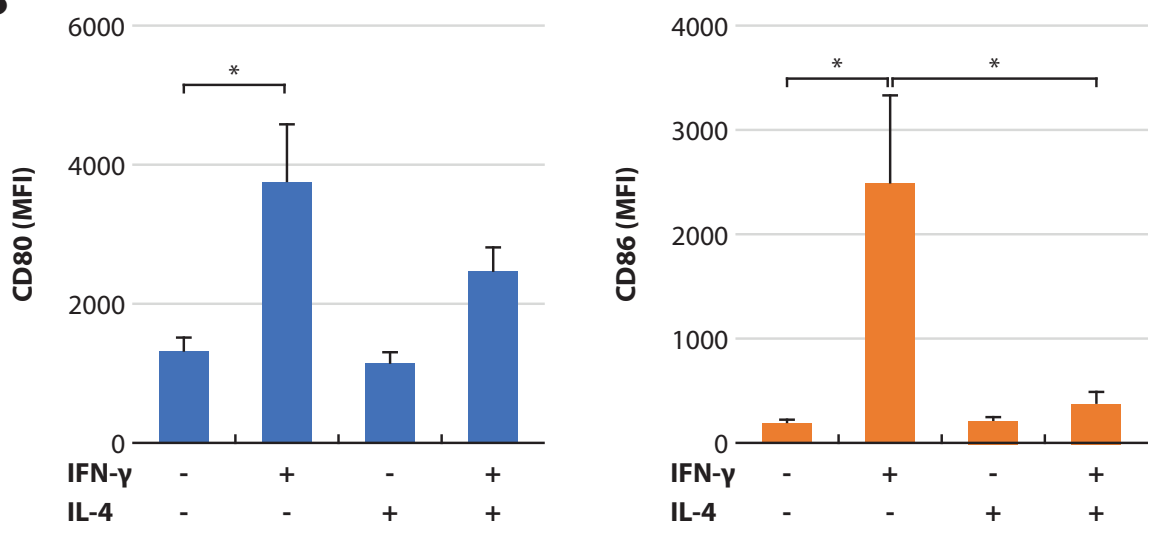

Figure 4. Effects of interferon (IFN)- $\gamma$ and interleukin (IL)-4 on cell surface expression of CD80 and CD86 in RAW264.7 macrophages. RAW264.7 macrophages were treated with IFN- $\gamma(40 \mathrm{ng} / \mathrm{ml})$, IL-4 (40 ng/ml), or both for 20 hours. Cell-surface expression of CD80 and CD86 was analyzed using flow cytometry. (A) Dot plots of CD80 and CD86 positive cells. (B) Mean fluorescence intensity of CD80 and CD86 expression. Each column represents the mean \pm standard error of the mean of four independent experiments. Statistical significance was calculated using Tukey's test $\left({ }^{*} p<0.05\right)$. 
A
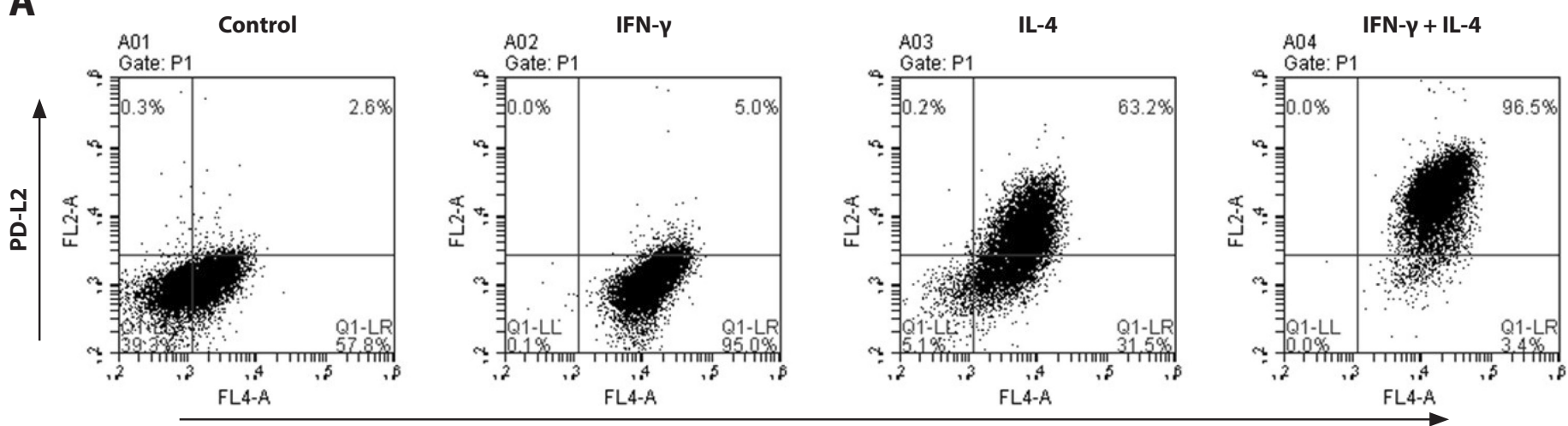

PD-L1

B

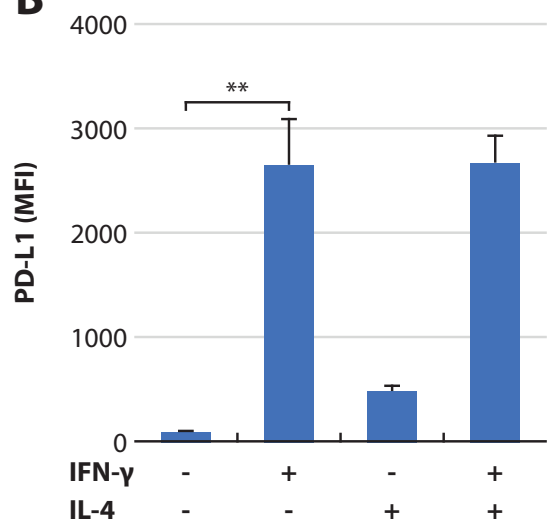

30000

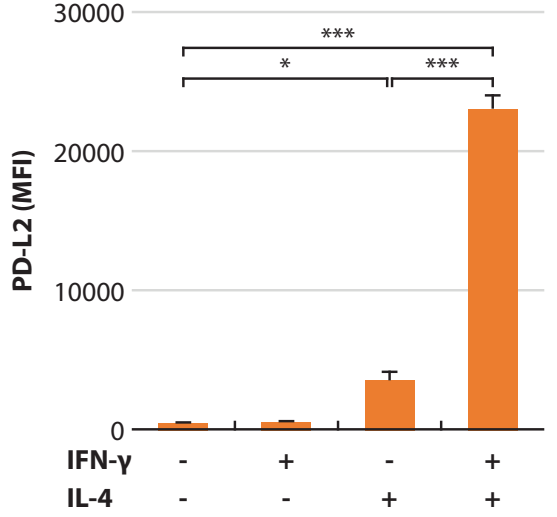

C

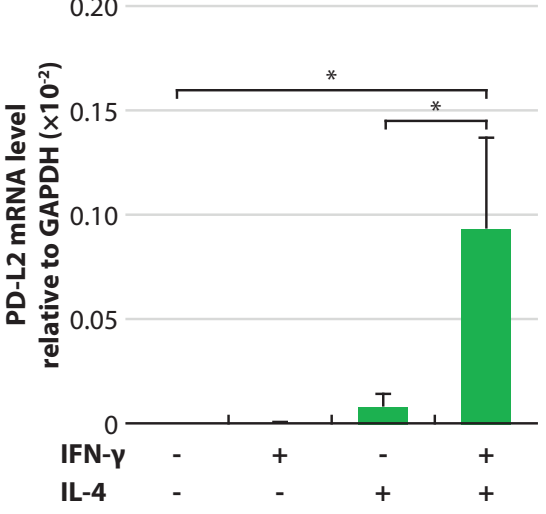

Figure 5. Effects of interferon (IFN)- $\gamma$ and interleukin (IL)-4 on cell surface expression of programmed death ligand (PDL)1 and PD-L2 in RAW264.7 macrophages. RAW264.7 macrophages were stimulated with IFN- $\gamma$ (40 ng/ml), IL-4 (40 ng/ $\mathrm{ml}$ ), or both. At 20 hours after the stimulation, the cell surface expression of PD-L1 and PD-L2 was analyzed using flow cytometry. (A) Dot plots of PD-L1 and PD-L2 positive cells. (B) Mean fluorescence intensity of PD-L1 and PD-L2 expression. Each column represents the mean \pm standard error of the mean of four independent experiments. (C) At 4 hours after the stimulation, the total RNA of the cells was isolated and the level of PD-L2 mRNA expression in the RNA samples was analyzed using quantitative reverse transcription-polymerase chain reaction. Each column represents the mean \pm standard error of the mean of ten independent experiments. Statistical significance was calculated using Tukey's test $\left(^{\star} p<0.05,{ }^{* *} p<0.01\right.$, $\left.{ }^{\star * *} p<0.001\right)$.

\section{STAT1 and STAT6 activation in RAW264.7 macrophages upon IFN- $\gamma$ and IL-4 stimulation}

Hematopoietic cytokine receptor signaling is largely mediated via the Janus kinase (JAK)-STAT pathway. ${ }^{21}$ STAT1 plays a major role in IFN- $\gamma$ signaling, while STAT6 is an essential mediator of IL-4 responses. We thus analyzed STAT1 and STAT6 activity in RAW264.7 macrophages upon IFN- $\gamma$ and IL-4 stimulation.

The cells were stimulated with IFN- $\gamma$, IL-4, or both for $30 \mathrm{~min}$ and intracellular protein levels of the active forms of STAT1 and STAT6, phospho-STAT1 (pSTAT1) and phospho-STAT6 (pSTAT6), were determined using immunoblotting (Figure 6). IFN- $\gamma$ markedly increased pSTAT1 expression in the cells, while exerting no influence on the level of pSTAT6. IL-4 induced a substantial level of pSTAT6, while showing no effect on the level of pSTAT1. Simultaneous stimulation with IFN- $\gamma$ and IL-4 resulted in the marked activation of both STAT1 and STAT6. It should be noted that IFN- $\gamma$ or IL-4 did not affect IL-4-induced STAT6 activation or IFN$\gamma$-induced STAT1 activation, respectively.

\section{Effect of IFN- $\gamma$ on IL4R mRNA expression}

It is possible that IFN- $\gamma$ affects IL4R expression. We thus examined the effect of IFN- $\gamma$ on mRNA expression of IL4R in RAW264.7 macrophages. The cells were stimulated with IFN- $\gamma$, IL- 4 , or both for 4,8 , and $16 \mathrm{hr}$ and the mRNA levels of IL4R in the cells were analyzed using quantitative RTPCR. IL-4 alone showed no effects on IL4R mRNA expression at the tested time points (Figure 6C). IFN- $\gamma$ alone markedly increased IL4R mRNA level at $4 \mathrm{hr}$, but its effect was not statistically significant ( $p=0.144$, Figure 6C left). In the presence of IL-4, IFN- $\gamma$ significantly increased IL4R mRNA level at the same time period. The IFN- $\gamma$-induced increase in the IL4R mRNA levels was attenuated at 8 and $16 \mathrm{hr}$ (Figure 6C center and right). 
A

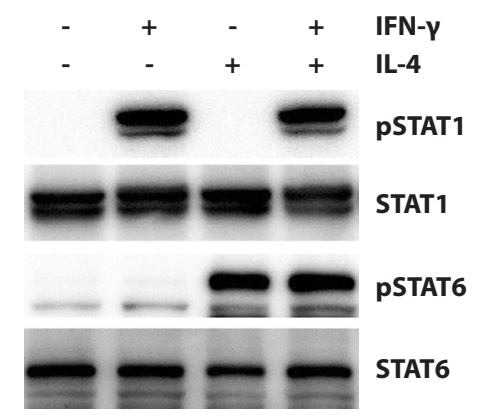

C

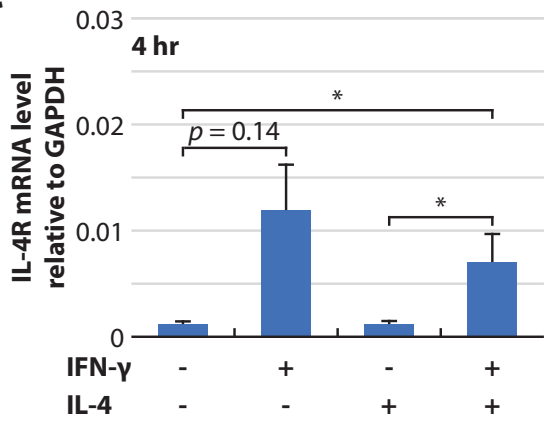

B
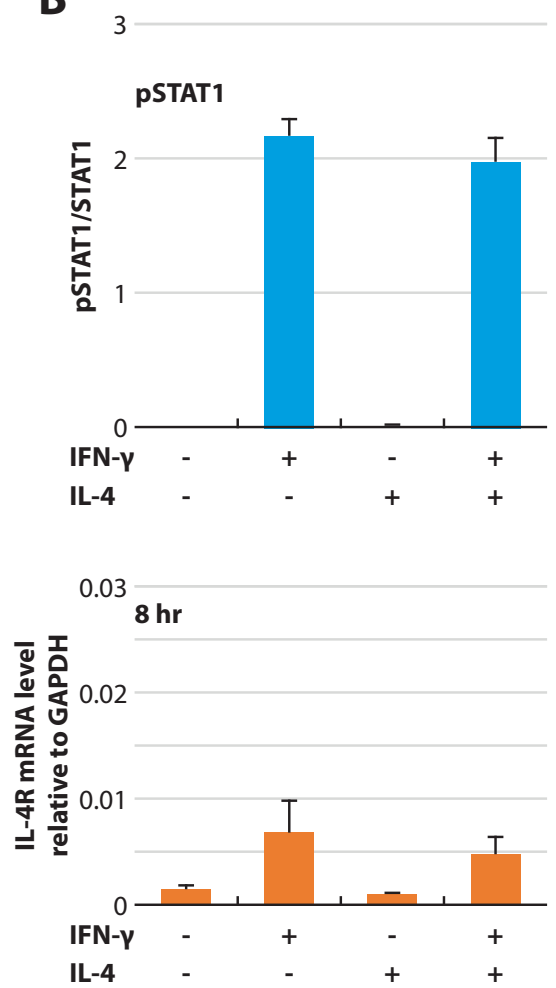
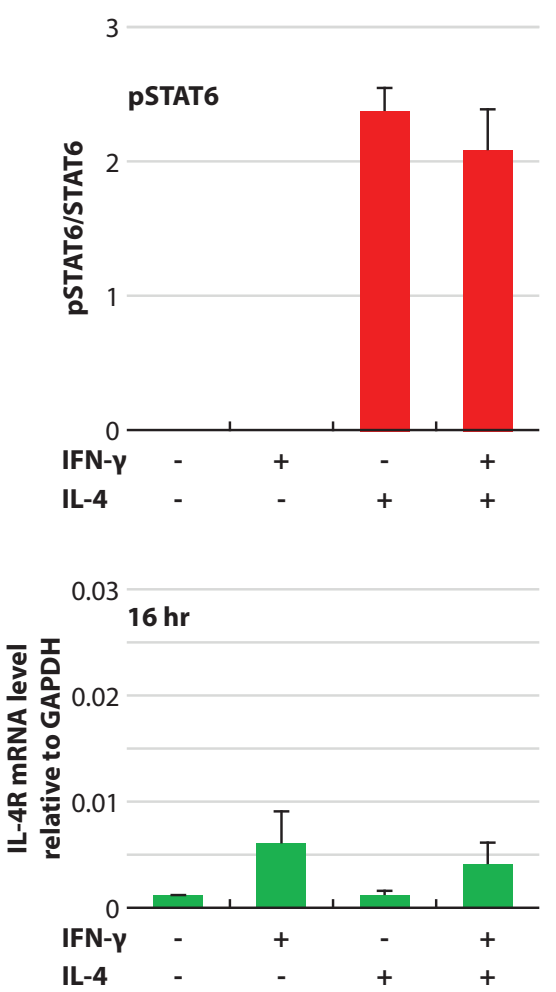

Figure 6. Signal transducers and activators of transcription (STAT) 1 and STAT6 activation in RAW264.7 macrophages upon interferon (IFN)- $\gamma$ and interleukin (IL)-4 stimulation. RAW264.7 macrophages were stimulated with IFN- $\gamma$ (40 ng/ml), IL-4 (40 ng/ml), or both for 30 minutes. The levels of phospho-STAT1, STAT1, phospho-STAT6, and STAT6 were determined using immunoblotting. (A) A representative immunoblot is shown. (B) The relative intensity of the specific band is shown. Each column represents the mean \pm standard error of the mean of seven (phospho-STAT1) or four (phospho-STAT6) independent experiments. (C) Effect of IFN- $\gamma$ on mRNA expression of IL-4 receptor a (IL4R). RAW264.7 macrophages were stimulated with IFN- $\gamma$, IL-4, or both for 4, 8, and 16 hours followed by total RNA isolation. The level of IL4R mRNA expression in the total RNA was analyzed using quantitative reverse transcription-polymerase chain reaction. Each column presents the mean \pm standard error of the mean of three independent experiments. Statistical significance was calculated using Tukey's test $\left({ }^{*} p<0.05\right)$.

\section{Discussion}

Th1/Th2 shift occurs in several immune disorders such as atopic dermatitis and contact hypersensitivity. ${ }^{22,23}$ Atopic dermatitis is considered a biphasic disease consisting of the Th2-mediated acute phase and the Th1-influencing chronic phase. Th2 cells are responsible for the pathogenesis of the acute phase via excessive production of Th2 cytokines, such as IL-4, IL-5, and IL-13, and inappropriate IgE synthesis, while Th1 cells seem to play a role in the chronic phase via Th1 cytokines including IFN- $\gamma \cdot{ }^{22}$ Both Th1 and Th2 immune pathways are enhanced in the lesional skin compared to that in the non-lesional skin in the chronic phase of atopic dermatitis. ${ }^{24}$ In contrast, Th1 immune responses are dominant in the lesional skin and the draining lymph nodes of the acute phase of contact hypersensitivity, whereas Th2 immune responses are induced in the chronic phase. ${ }^{24} \mathrm{~A}$ shift from Th1 to Th2 is also observed in experimental autoimmune encephalomyelitis and patients with multiple sclerosis. ${ }^{25,26}$ Therefore, Th1 and Th2 cytokines appear to coexist in the chronic phase of immune disorders. However, the combined action of Th1 and Th2 cytokines is not fully understood. In this study, we examined the effect of simultaneous treatment with representative
Th1 and Th2 cytokines, IFN- $\gamma$ and IL-4, on RAW264.7 macrophage functions and found that these cytokines together synergistically increased Arg-1 and PD-L2 expression. Arg-1 is responsible for tissue repair and anti-inflammation, ${ }^{15}$ while PD-L2 plays a role in $T$ cell suppression. ${ }^{19,27}$ Macrophages highly expressing Arg-1 and PD-L2 may be induced in the chronic phase of immune disorders coexistence with IFN- $\gamma$ and IL-4 to inhibit prolonged inflammatory immune responses.

PD-L1 and PD-L2, which are ligands for PD-1 expressed on $\mathrm{T}$ cells, are responsible for the inhibition of $\mathrm{T}$ cell activation. ${ }^{19}$ PD-L1 is constitutively expressed in various types of immune- and non-immune cells and can be upregulated by inflammatory stimuli. ${ }^{19,28,29} \mathrm{PD}-\mathrm{L} 2$ is predominantly expressed in antigen-presenting cells, such as dendritic cells and macrophages, and the constitutive basal level is low. It has been reported that IFN- $\gamma$ plus LPS stimulation significantly increases macrophage expression of PD-L1 but not PD-L2. ${ }^{30}$ In contrast, IL-4 markedly increases PD-L2 expression on peritoneal macrophages, while showing modest effect on PD-L1 expression. It has been suggested that PD-L1 and PD-L2 are differently 
regulated by Th1 and Th2 cytokines. Although it has been demonstrated that IL-4 induces PD-L2 expression in macrophages, none of studies report the synergy of IL- 4 and IFN- $\gamma$ in PD-L2 expression. Conversely, PD-L1 ${ }^{+} \mathrm{PD}-\mathrm{L} 2^{+}$alternatively activated macrophages were generated in Taenia crassiceps-infected mice and exhibited anti-inflammatory properties. ${ }^{31}$ In this study, we found that IFN- $\gamma$ and IL-4 synergistically increased PD-L2 expression in RAW264.7 macrophages, resulting in the generation of PD-L1 and PD-L2 highly positive cells. In contrast, these cells showed a decrease in the expression of inflammatory cytokines and CD86. Therefore, this type of macrophage might play a role in immune suppression.

It has been reported that IL-4-induced expression of Arg1 and PD-L2 is dependent on the STAT6-mediated pathway, ${ }^{20}$ raising the possibility that IL- 4 and IFN $-\gamma$ cooperatively activate STAT6 in RAW264.7 macrophages. However, STAT6 activity with IL-4 plus IFN- $\gamma$ was similar to that with IL-4 alone, suggesting that signaling pathway(s) other than STAT6 may be responsible for the synergy in Arg-1 and PD-L2 production. Previous studies have suggested that mitogen-activated protein kinases and phosphoinositide-3-kinase play a role in IL-4-induced Arg-1 expression. ${ }^{32,33}$ Thus, we also examined the activity of extracellular signal-regulated kinase 1/2, p38 mitogen-activated protein kinase, c-jun $\mathrm{N}$-terminal kinase, and Akt, a downstream effector of phosphoinositide-3-kinase, upon IL-4 and IFN- $\gamma$ stimulation. However, we could not detect the combined effect of IL- 4 and IFN- $\gamma$ in the activity of these signaling molecules (data not shown).

Recently, it was suggested that IL-4R-mediated ERK5 signaling regulates macrophage expression of Arg-1 in a STAT6-independent manner. ${ }^{34}$ At present, we found that IFN- $\gamma$ with or without IL- 4 increased IL-4R expression in RAW264.7 macrophages. Therefore, we speculated that IL4R-mediated ERK5 signaling and/or unknown pass way(s) are enhanced by the increase in IL- $4 \mathrm{R}$, and thereby Arg- 1 and PD-L2 expression was markedly induced in the presence of IL-4 and IFN- $\gamma$. Anyway, the molecular mechanism underlying the synergy of IL- 4 and IFN- $\gamma$ on Arg-1 and PD-L2 expression should be carefully analyzed in future studies.

Piccolo et $\mathrm{al}^{35}$ showed that IFN- $\gamma$ and IL-4 mutually inhibited epigenomic and transcriptional changes induced by each cytokine alone in mouse bone marrow derived macrophages (BMDMs). Although the mutual antagonistic effects of these cytokines were well discussed, their cooperative action like a synergy was less well documented. In their study, IFN- $\gamma$ partially attenuated IL-4-induced Arg- 1 mRNA expression. In contrast, we found that IFN- $\gamma$ synergistically enhanced IL4-induced Arg-1 expression at mRNA and protein levels. It seems to us that this discrepancy is attributable to the difference of macrophage origin and/or culture condition. BMDMs were prepared by culturing with L929-conditioned medium containing M-CSF for 6 days. The L929-conditioned medium appears to contain unknown factors other than M-CSF. These factors might affect the regulation of Arg-1 mRNA expression.

In the present study, we showed the synergy of IFN- $\gamma$ and IL-4 in Arg-1 and PD-L2 expression. This regulation may contribute to the suppression of excessive undesirable inflammation in the chronic phase of inflammatory diseases, in which IFN- $\gamma$ and IL- 4 coexist. The elucidation of the precise mechanism underlying the synergy of IFN- $\gamma$ and IL-4 may lead to the development of new strategies for the treatment of inflammatory immune disorders.

\section{Conclusions}

Cooperative action of IFN- $\gamma$ and IL- 4 is not fully understood. This study showed synergy of IFN- $\gamma$ and IL-4 in Arg-1 and PD-L2 expression in RAW264.7 macrophages. Arg-1 and PD-L2 are responsible for anti-inflammation and immune suppression. Thus, macrophages highly expressing Arg-1 and PD-L 2 may be induced by both IFN- $\gamma$ and IL-4 at the inflammatory site, and might play a role in the regulation of inflammatory immune responses.

\section{Conflict of interest}

The authors have no conflict of interest to disclose.

\section{References}

1. Vahedi G, Poholek AC, Hand TW, Laurence A, Kanno Y, O'Shea JJ, et al. Helper T-cell identity and evolution of differential transcriptomes and epigenomes. Immunol Rev. 2013;252:24-40.

2. Raphael I, Forsthuber TG. Stability of T-cell lineages in autoimmune diseases. Expert Rev Clin Immunol. 2012;8:299-301.

3. Skurkovich S, Skurkovich B. Anticytokine therapy, especially anti -interferon- $\gamma$, as a pathogenetic treatment in TH-1 autoimmune diseases. Ann N Y Acad Sci. 2005;1051:684-700.

4. Ferber IA, Brocke S, Taylor-Edwards C, Ridgway W, Dinisco C, Steinman $\mathrm{L}$, et al. Mice with a disrupted IFN- $\gamma$ gene are susceptible to the induction of experimental autoimmune encephalomyelitis (EAE). J Immunol. 1996;156:5-7.

5. Vermeire K, Heremans H, Vandeputte M, Huang S, Billiau A, Matthys P. Accelerated collagen-induced arthritis in IFN- $\gamma$ receptor-deficient mice. J Immunol. 1997;158:5507-13.

6. Manoury-Schwartz B, Chiocchia G, Bessis N, Abehsira-Amar O, Batteux F, Muller S, et al. High susceptibility to collagen-induced arthritis in mice lacking IFN- $\gamma$ receptors. J Immunol. 1997;158:5501-6.

7. Akdis CA, Arkwright PD, Brüggen MC, Busse W, Gadina M, Guttman -Yassky E, et al. Type 2 immunity in the skin and lungs. Allergy. 2020; 75:1582-1605.

8. Levings MK, Schrader JW. IL-4 inhibits the production of TNF- $\alpha$ and IL-12 by STAT6-dependent and -independent mechanisms. J Immunol. 1999;162:5224-9.

9. Mullen AC, Hutchins AS, High FA, Lee HW, Sykes KJ, Chodosh LA, et al. Hlx is induced by and genetically interacts with T-bet to promote heritable TH1 gene induction. Nat Immunol, 2002;3:652-8.

10. Djuretic IM, Levanon D, Negreanu V, Groner Y, Rao A, Ansel KM. Transcription factors T-bet and Runx3 cooperate to activate Ifng and silence Il4 in T helper type 1 cells. Nat Immunol 2007;8:145-53.

11. Shapouri-Moghaddam A, Mohammadian S, Vazini H, Taghadosi M, Esmaeili SA, Mardani F, et al. Macrophage plasticity, polarization, and function in health and disease. J Cell Physiol. 2018;233:6425-40.

12. Murray PJ, Allen JE, Biswas SK, Fisher EA, Gilroy DW, Goerdt S, et al. Macrophage activation and polarization: nomenclature and experimental guidelines. Immunity. 2014;41:14-20.

13. Grailer JJ, Haggadone MD, Sarma JV, Zetoune FS, Ward PA. Induction of $\mathrm{M} 2$ regulatory macrophages through the $\beta_{2}$-adrenergic receptor with protection during endotoxemia and acute lung injury. J Innate Immun. 2014;6:607-18.

14. Carneros D, Medina-Gómez G, Giralt M, León-Camacho M, Campbell M, Moreno-Aliaga MJ, et al. Cardiotrophin-1 is an anti-inflammatory cytokine and promotes IL-4-induced M2 macrophage polarization. FASEB J. 2019;33:7578-87.

15. Campbell L, Saville CR, Murray PJ, Cruickshank SM, Hardman MJ. Local arginase 1 activity is required for cutaneous wound healing. J Invest Dermatol. 2013;133:2461-70. 
16. Ishikawa S, Saito Y, Yanagawa Y, Otani S, Hiraide S, Shimamura K, et al. Early postnatal stress alters extracellular signal-regulated kinase signaling in the corticolimbic system modulating emotional circuitry in adult rats. Eur J Neurosci. 2012;35:135-45.

17. Yanagawa $\mathrm{Y}$, Matsumoto $\mathrm{M}$, Togashi H. Adrenoceptor-mediated enhancement of interleukin-33 production by dendritic cells. Brain Behav Immun. 2011;25:1427-33.

18. Chambers CA, Allison JP. Costimulatory regulation of $\mathrm{T}$ cell function. Curr Opin Cell Biol. 1999;11:203-10.

19. Liang L, Sha WC. The right place at the right time: novel B7 family members regulate effector T cell responses. Curr Opin Immunol. 2002;14:384-90.

20. Huber S, Hoffmann R, Muskens F, Voehringer D. Alternatively activated macrophages inhibit T-cell proliferation by Stat6-dependent expression of PD-L2. Blood. 2010;116:3311-20.

21. Baker SJ, Rane SG, Reddy EP. Hematopoietic cytokine receptor signaling. Oncogene. 2007;26:6724-37.

22. Grewe M, Bruijnzeel-Koomen CA, Schöpf E, Thepen T, Langeveld -Wildschut AG, Ruzicka T, Krutmann J. A role for Th1 and Th2 cells in the immunopathogenesis of atopic dermatitis. Immunol Today. 1998;19: $359-61$.

23. Kitagaki H, Kimishima M, Teraki Y, Hayakawa J, Hayakawa K, Fujisawa $S$, et al. Distinct in vivo and in vitro cytokine profiles of draining lymph node cells in acute and chronic phases of contact hypersensitivity: importance of a type 2 cytokine-rich cutaneous milieu for the development of an early-type response in the chronic phase. J Immunol. 1999;163: $1265-73$.

24. Gittler JK, Shemer A, Suárez-Fariñas M. Progressive activation of TH2/ TH22 cytokines and selective epidermal proteins characterizes acute and chronic atopic dermatitis. J Allergy Clin Immunol. 2012;130:1344-54.

25. Genain CP, Abel K, Belmar N, Villinger F, Rosenberg DP, Linington C, et al. Late complications of immune deviation therapy in a nonhuman primate. Science. 1996;274:2054-7.
26. Antel JP, Bar-Or A. Do myelin-directed antibodies predict multiple sclerosis? N Engl J Med. 2003;349:107-9.

27. Solinas C, Aiello M, Rozali E, Lambertini M, Willard-Gallo K, Migliori E. Programmed cell death-ligand 2: A neglected but important target in the immune response to cancer? Transl Oncol 2020;13:100811.

28. Matzinger P, Kamala T. Tissue-based class control: the other side of tolerance. Nat Rev Immunol. 2011;11:221-30.

29. Rozali EN, Hato SV, Robinson BW, Lake RA, Lesterhuis WJ. Programmed death ligand 2 in cancer-induced immune suppression. Clin Dev Immunol 2012;656340.

30. Loke P, Allison JP. PD-L1 and PD-L2 are differentially regulated by Th1 and Th2 cells. Proc Natl Acad Sci USA. 2003;100: 5336-41.

31. Terrazas C, de Dios Ruiz-Rosado J, Amici SA, Jablonski KA, Martinez-Saucedo D, Webb LM, Cortado H, et al. Helminth-induced Ly6Chi monocyte-derived alternatively activated macrophages suppress experimental autoimmune encephalomyelitis. Sci Rep. 2017;7:40814.

32. Jiménez-Garcia L, Herránz S, Luque A, Hortelano S. Critical role of p38 MAPK in IL-4-induced alternative activation of peritoneal macrophages. Eur J Immunol. 2015;45:273-86.

33. Hao J, Hu Y, Li Y, Zhou Q, Lv X. Involvement of JNK signaling in IL4-induced M2 macrophage polarization. Exp Cell Res. 2017;357:155-62.

34. Luiz JPM, Toller-Kawahisa JE, Viacava PR, Nascimento DC, Pereira PT, Saraiva AL, et al. MEK5/ERK5 signaling mediates IL-4-induced M2 macrophage differentiation through regulation of c-Myc expression. J Leukoc Biol. 2020;108:1215-23.

35. Piccolo V, Curina A, Genua M, Ghisletti S, Simonatto M, Sabò A, et al. Opposing macrophage polarization programs show extensive epigenomic and transcriptional cross-talk. Nat Immunol. 2017;18:530-40. 